\title{
Simplification des procédures TAVI : mise au point
}

\section{TAVI simplification: a focus}

M. Akodad ${ }^{1,2} \mathrm{MD}, \mathrm{T}$. Lefèvre ${ }^{3} \mathrm{MD}, \mathrm{Y}$. Lounes $\mathrm{MD}^{4}, \mathrm{~F}$. Leclercq ${ }^{1,2} \mathrm{MD}, \mathrm{PhD}$

${ }^{1}$ Service de cardiologie, centre hospitalier universitaire Arnaud de Villeneuve, Montpellier, France

${ }^{2}$ PhyMed Exp, Université de Montpellier, INSERM U1046, CNRS UMR 9214, 34295 Montpellier cedex 5, France

${ }^{3}$ Ramsay Générale de Santé, Institut Cardiovasculaire Paris Sud, Massy, France

${ }^{4}$ Service de chirurgie vasculaire, centre hospitalier universitaire Arnaud de Villeneuve, Montpellier, France

Adresse de correspondance:

Mariama Akodad

Service de cardiologie

Centre hospitalier universitaire Arnaud de Villeneuve,

371 avenue du doyen Gaston Giraud

34090 Montpellier, France

E-mail : akodadmyriam@gmail.com

$\underline{\text { Tél: }+33467332501}$

Fax: +33467336230 


\section{Résumé}

Depuis la première procédure réalisée en 2002 par Alain Cribier, le remplacement valvulaire aortique percutanée ou TAVI a révolutionné la prise en charge du rétrécissement aortique avec une augmentation constante du nombre de procédures réalisées à travers le monde. L'évolution de la technique et du matériel associée à l'expérience grandissante des opérateurs et des équipes se sont accompagnées d'une diminution drastique des complications chez des patients à risque chirurgical de moins en moins important. En parallèle, la procédure s'est considérablement simplifiée, réalisée de plus en plus sous anesthésie loco-régionale, avec abord fémoral percutané, abord radial secondaire, implantation de la prothèse sans prédilatation, stimulation sur guide placé dans le ventricule gauche et sortie précoce du patient. Ainsi, le TAVI « simplifié », adopté dans la plupart des centres de nos jours, est une véritable révolution de la technique originelle. Cependant, proposer un TAVI simplifié doit obligatoirement s'accompagner en amont d'une sélection rigoureuse des patients pouvant bénéficier d'une procédure minimaliste afin d'en garantir la sécurité.

Mots-clés: TAVI, simplification, minimaliste, parcours de soins, sortie précoce 


\section{Abstract}

After a first procedure carried out in 2002 by Pr Cribier's, Transcatheter Aortic Valve Replacement or TAVR revolutionized the management of aortic stenosis with a constant increase in the number of procedures performed worldwide. Experience of operators and teams and evolution of the technique has been accompanied by a drastic reduction in complications in patients at lower surgical risk. In parallel, the procedure was considerably simplified, carried out more and more under local anesthesia, with percutaneous femoral approach, secondary radial approach, prosthesis implantation without predilatation, rapid pacing on left ventricle wire and early discharge. Thus, the "simplified" TAVR adopted in most centers nowadays is a real revolution of the technique. However, simplified TAVR must be accompanied upstream by a rigorous selection of patients who can benefit from a minimalist procedure in order to guarantee its safety.

Key words: TAVI, simplification, minimalist, pathway, early discharge 


\section{Introduction}

Depuis la première intervention réalisée avec succès en 2002 [1], le remplacement valvulaire aortique percutanée (TAVI) s'est largement répandu à travers le monde avec plus de 500000 implantations réalisées à ce jour dans plus de 85 pays [2]. Dans ce contexte, les indications ont récemment été étendues aux patients de plus de 75 ans à risque chirurgical modéré en cas d'accès transfémoral favorable après discussion en "heart team » [3]. De plus, deux études randomisées incluant des patients à bas risque chirurgical ont montré une noninfériorité de la technique percutanée par rapport à la chirurgie avec des faibles taux de complications [4,5]. Cette diminution drastique des complications est liée à plusieurs éléments. En effet, les améliorations techniques des prothèses de dernière génération, l'amélioration du profil des nouveaux dispositifs, l'expérience croissante des opérateurs et la meilleure sélection des patients ont ensemble contribué à améliorer les résultats de la technique. Celle-ci a donc pu évoluer vers une procédure de plus en plus simplifiée à chaque étape. Ainsi l'anesthésie locale, l'accès radial controlatéral, l'absence de prédilatation systématique et la stimulation sur le guide ventriculaire gauche (VG) simplifient considérablement la procédure et contribueraient à réduire les complications [6]. Plus récemment, plusieurs équipes ont également proposé des algorithmes de simplification du parcours de soins en post-procédure permettant une réduction de la durée d'hospitalisation pour un certain nombre de patients avec un bénéfice médico-économique évident $[7,8]$. Ainsi, le TAVI « simplifié » prend de l'ampleur et est adopté dans la plupart des centres de nos jours chez certains patients sélectionnés. Nous allons décrire, dans cette revue, la sélection des patients et la planification d'une procédure simplifiée, les étapes clés d'un TAVI simplifié et exposer les données de la littérature concernant l'impact de cette simplification sur les résultats de la technique. 


\section{Sélection des patients et planification de la procédure}

L'une des étapes clés avant d'envisager une procédure TAVI est l'évaluation précise du profil de risque du patient intégrant les comorbidités ainsi que l'évaluation morphologique par scanner permettant d'anticiper d'éventuelles complications. Cette étape permet également de sélectionner les candidats potentiels à une procédure et à un parcours de soins simplifiés (Figure 1). Tout d'abord, l'évaluation de la faisabilité d'un abord transfémoral, possible dans plus de $90 \%$ des cas [9], est indispensable avant d'envisager une procédure simplifiée. De même l'anatomie valvulaire aortique, la présence de calcifications importantes, la possibilité d'un accès radial et les éléments en faveurs d'un risque accru de troubles conductifs pendant ou au décours immédiat de la procédure doivent être évalués rigoureusement [6].

Cette simplification de la procédure peut ainsi être anticipée avant l'hospitalisation pour la procédure TAVI et être exposée au patient et à son entourage [7] après décision de la « Heart Team ", équipe composée de cardiologues interventionnels, cardiologues non interventionnels, gériatres, anesthésistes et coordinateurs médicaux et/ou paramédicaux (Figure 2). En cas d'approche simplifiée, la procédure peut être réalisée en routine par deux opérateurs, deux infirmières et un anesthésiste. Un chirurgien cardiaque et un échocardiographiste ne sont pas obligatoirement présents dans la salle, mais doivent être disponibles[6].

\section{Eléments de simplification}

Le TAVI simplifié débute dès l'installation du patient avec le choix du type d'anesthésie jusqu'à la sortie d’hospitalisation, précoce ou non (Figure 3).

Le schéma type d'une procédure simplifiée inclut une anesthésie loco-régionale, un abord transfémoral percutané, un abord radial controlatéral, l'absence de prédilatation et la stimulation sur guide VG afin d'éviter une voie d'abord veineuse fémorale. En post-procédure, 
le parcours de soins peut être simplifié par l'absence d'hospitalisation systématique en soins intensifs et la sortie précoce (Figure 3) [6].

\section{Anesthésie locorégionale et sédation consciente}

Dans la première expérience du TAVI, la procédure était généralement réalisée sous anesthésie générale avec monitoring lourd (cathéter veineux central, cathéter artériel, intubation orotrachéal, sondage urinaire), et ce type d'anesthésie reste d'ailleurs encore largement utilisée en Amérique du Nord [10]. L'une des premières étapes à être simplifiée a été celle de l'anesthésie avec l'avènement des procédures réalisées sous anesthésie locorégionale seule ou avec sédation consciente. La faisabilité a en fait été décrite dès 2002 puisque cette première procédure était réalisée sous anesthésie locorégionale[1]. L'anesthésie locorégionale avec sédation consciente, consistant en une infiltration du site d'accès vasculaire par la lidocaïne et une administration intraveineuse d'agents analgésiques, a été progressivement adoptée par plusieurs équipes et concernait plus de $60 \%$ des patients du registre France 2 [11].

\section{Abord fémoral percutané}

La réduction du diamètre des cathéters, de 24 French initialement à 14 French avec les prothèses de dernière génération, a largement contribué à l'avènement des techniques percutanées de "préclosing " de l'artère fémorale venant remplacer l'abord chirurgical. L'approche percutanée est réalisée principalement avec les dispositifs de fermeture, Prostar et Proglide (Abbott Vascular Devices, Redwood City, Californie, États-Unis) [12]. Plus que le type de dispositif percutané, la technique percutanée doit être rigoureuse pour limiter les complications au site d'accès. En effet, le site de ponction de l'artère fémorale commune doit être sélectionné sur le scanner d'angiographie avant la procédure dans une zone sans calcium et au-dessus de la bifurcation fémorale. Au cours de la procédure, la ponction doit être 
effectuée sous guidage angiographique ou par ultrasons au centre de la paroi artérielle antérieure [6]. Les dispositifs de fermeture percutanés sont ensuite déployés comme décrit précédemment [12]. De nouveaux dispositifs ont vu le jour plus récemment avec notamment le système MANTA avec un plug de collagène appliqué de part et d'autre du point de ponction fémoral [13].

\section{L'abord radial comme accès secondaire}

L'accès radial comme voie d'abord secondaire, abord permettant de réaliser les angiographies de repérage et de contrôle au cours de la procédure TAVI, tend à remplacer peu à peu l'abord fémoral. En effet, bien que les complications vasculaires aient considérablement diminué au fil du temps, un tiers des complications vasculaires majeures surviennent encore au niveau de l'accès fémoral controlatéral [14]. La sélection d'un accès radial comme voie d'abord secondaire, décrite précédemment, apparaît alors séduisante pour réduire les complications vasculaires $[15,16]$. Les limites de cette voie d'abord comprennent le spasme qui peut gêner la mobilisation des cathéters et dans quelques rares cas, une conversion nécessaire vers une approche fémorale controlatérale en cross-over, en particulier en cas de nécessité d'implantation de stent couvert lors de la gestion de complication vasculaire sur la voie d'abord principale.

\section{Direct TAVI}

Lors de l'expérience initiale du TAVI, la prédilatation au ballon était systématique afin de permettre le passage et d'optimiser le déploiement de la prothèse. Cependant, la réalisation de TAVI sans prédilatation a été évaluée pour la première fois en 2011 par Grübe et al. [17] et s'est avérée réalisable dans des études non randomisées $[18,19]$. Actuellement, avec les dispositifs de nouvelle génération ayant un meilleur profil de franchissement, la prédilatation 
au ballon ne semble pas indispensable avant l'implantation de la prothèse qu'elle soit « balloon-expandable» ou auto expansible et cette approche en « direct TAVI » a été adoptée par de nombreux centres [20]. Cependant, une prédilation doit probablement être envisagée dans les cas de sténose aortique sévèrement calcifiée avec une surface aortique $<0,5 \mathrm{~cm} 2$, afin de réduire le risque de sous-expansion de la prothèse et le besoin de post-dilater, en particulier avec les prothèses auto expansibles [19].

Stimulation sur guide VG

Lors du déploiement des valves montées sur ballon, une stimulation ventriculaire rapide est obligatoire dans le but de provoquer une hypotension sévère transitoire évitant ainsi une embolisation de la prothèse. La stimulation rapide était effectuée de façon systématique par l'implantation d'une sonde de stimulation temporaire dans le ventricule droit jusque très récemment, par voie veineuse le plus souvent fémorale [21]. Cependant, cette technique peut être associée à la survenue de complications vasculaires au site de ponction ou de perforation du ventricule droit par la sonde avec risque de tamponnade [21]. Récemment, une stimulation ventriculaire rapide à travers le guide positionné dans le VG a été décrite dans le but de simplifier la procédure en supprimant un accès vasculaire supplémentaire lors de la valvuloplastie aortique au ballon ou du $\operatorname{TAVI}[6,21]$. La stimulation est réalisée à l'aide de pinces crocodiles, la pince noire est raccordée au guide (anode) et la pince rouge est raccordée à la peau via une aiguille métallique (cathode). Le stimulateur est réglé en amplitude maximale et détection minimale et le guide doit être isolé par un cathéter ou le système de délivrance de la valve.

Simplification du parcours de soins

Un autre aspect concerne la simplification des suites de la procédure avec un lever précoce 
dès H6 [9] et un parcours de soins simplifié avec notamment un passage non systématique en soins intensifs et un raccourcissement de la durée d'hospitalisation chez certains patients sélectionnés [7, 8, 22]. En post-procédure TAVI, une surveillance rapprochée est systématique dans le but de détecter les complications, survenant pour la plupart dans les heures qui suivent la procédure [23]. Ainsi, l'admission non systématique en unité de soins intensifs après la procédure a été évaluée et s'est avérée faisable et sûre chez certains patients sélectionnés notamment sur le faible risque de troubles conductifs, après une évaluation rigoureuse et surveillance de 2 heures en salle de réveil [22]. En effet, l'un des risques majeurs à anticiper est le risque de trouble de la conduction retardé, avec un risque nul dans l'étude de Toggweiller et al., chez les patients avec un ECG de base normal et l'absence de trouble conductif en post-procédure immédiat [24]. De même, la sortie précoce chez certains patients sélectionnés est un autre élément du TAVI simplifié. Plusieurs équipes ont récemment proposé des algorithmes permettant d'anticiper un raccourcissement de la durée $d^{\prime}$ hospitalisation selon des critères prédéfinis $[8,25]$. Ainsi avec l'augmentation constante du nombre de procédures et la nécessité de répondre aux besoins en termes de structures d'aval, le raccourcissement de la durée d'hospitalisation reste un objectif prioritaire.

\section{Impact du TAVI simplifié dans la littérature}

\section{Anesthésie locorégionale et sédation consciente}

Aucune étude randomisée n'a été publiée comparant les évènements cliniques du TAVI en fonction du type d'anesthésie. Cependant, plusieurs registres rapportent un risque accru d'instabilité hémodynamique, une augmentation des pneumopathies, une extubation difficile et une identification tardive des complications comme l'accident vasculaire cérébral ou la dissection aortique chez les patients sous anesthésie générale [26, 27]. Dans deux méta- 
analyses récente comparant anesthésie générale et locorégionale avec sédation consciente, les résultats des deux approches étaient similaires en termes d'évènements majeurs mais avec des besoins en catécholamines et en transfusions moins fréquents ainsi qu'une durée d'hospitalisation plus courte chez les patients sous anesthésie locorégionale [28,29]. Cependant, dans ces méta-analyses, environ $6 \%$ ont nécessité une conversion de l'anesthésie locorégionale à l'anesthésie générale en raison de complications procédurales. Ainsi, les données de registre ont démontré la faisabilité et la sécurité et la de l'anesthésie locorégionale dans les procédures TAVI avec un impact médico-économique potentiel.

\section{Accès vasculaires}

Bien que l'approche chirurgicale soit associée à un faible taux de complications vasculaires dans la littérature [30], la fermeture percutanée de l’abord fémoral est considérée comme une technique moins invasive. Les dernières données rapportent également des taux de complications vasculaires très faibles après abord percutané [4,5]. De plus, l'accès percutané peut être associé à une durée d'hospitalisation plus courte [31]. De même, l'approche radiale s'est avérée faisable et sûre avec réduction des complications vasculaires lors des interventions percutanées et de la procédure TAVI en particulier [14-16]. Cependant, aucune étude randomisée n'est actuellement disponible comparant l'approche percutanée versus chirurgicale et l'utilisation de la voie radiale versus la voie fémorale en approche secondaire.

\section{Direct TAVI}

Dans la littérature, la prédilatation pourrait présenter quelques inconvénients comme un risque accru d'embolisation cérébrale ou la survenue d'une fuite aortique massive mal tolérée sur le plan hémodynamique [17-20]. Plus récemment, la non-infériorité du Direct TAVI a été démontrée avec la valve auto-expandable [32]. Une étude évaluant la non-inferiorité du TAVI 
sans prédilatation systématique avec la valve de nouvelle génération Sapien 3 a récemment terminé ses inclusions [33] (NCT02729519).

\section{Stimulation sur guide VG}

Peu de données sont disponibles dans la littérature évaluant l'impact de cette nouvelle technique sur la survenue de complications. Un seul essai randomisé comparant les deux techniques de stimulation a récemment terminé ses inclusions (NCT02781896).

\section{Simplification du parcours de soins}

De même que l'hospitalisation non systématique en soins intensifs a été démontrée comme faisable de façon sécuritaire chez environ $30 \%$ des patients [22], I'innocuité d'une sortie précoce a été démontrée chez certains patients sélectionnés, hospitalisés moins de 48 heures $[8,34]$. En effet, la durée médiane d'hospitalisation était d'un jour dans le groupe de sortie précoce, sans différence entre la sortie précoce et la sortie $>48$ heures en termes de mortalité, d'accident vasculaire cérébral et de réadmission à 1 mois. Une procédure de TAVI « minimaliste » avec anesthésie locale, moins de prédilatation au ballon et moins de monitoring invasif permettait de prédire une sortie précoce dans cette étude [8, 34].

\section{Conclusion}

Le TAVI « simplifié » a déjà été adopté en routine dans les centres expérimentés avec un taux de complications faible, un temps de procédure plus court, un meilleur confort du patient, une réduction des coûts et une diminution de la charge de travail pour le personnel. Cependant, une sélection rigoureuse des patients et une stratification précise du risque de complications sont la clé pour assurer la sécurité d'une procédure minimaliste. Plusieurs essais randomisés visent à évaluer chaque élément de cette approche TAVI simplifiée afin de garantir 
sa faisabilité avant l'extension des indications, probablement à venir, chez des patients à moindre risque. 


\section{Références}

[1] Cribier A, Eltchaninoff H, Bash A, Borenstein N, Tron C, Bauer F, et al. Percutaneous transcatheter implantation of an aortic valve prosthesis for calcific aortic stenosis: first human case description. Circulation. 2002 Dec 10;106(24):3006-8.

[2] Benamer H, Auffret V, Cayla G, Chevalier B, Dupouy P, Eltchaninoff H, et al. Position paper of French Interventional Group (GACl) for TAVI in France in 2018. Ann Cardiol Angeiol (Paris). 2018 Dec;67(6):455-65.

[3] Baumgartner H, Falk V, Bax JJ, De Bonis M, Hamm C, Holm PJ, et al. 2017 ESC/EACTS Guidelines for the management of valvular heart disease. Eur Heart J. 2017 Sep 21;38(36):2739-91.

[4] Mack MJ, Leon MB, Thourani VH, Makkar R, Kodali SK, Russo M, et al. Transcatheter Aortic-Valve Replacement with a Balloon-Expandable Valve in Low-Risk Patients. N Engl J Med. 2019 02;380(18):1695-705.

[5] Popma JJ, Deeb GM, Yakubov SJ, Mumtaz M, Gada H, O'Hair D, et al. Transcatheter Aortic-Valve Replacement with a Self-Expanding Valve in Low-Risk Patients. New England Journal of Medicine. 2019 May 2;380(18):1706-15.

[6] Akodad M, Lefèvre T. TAVI: Simplification Is the Ultimate Sophistication. Front Cardiovasc Med. 2018;5:96.

[7] Durand E, Eltchaninoff H, Canville A, Bouhzam N, Godin M, Tron C, et al. Feasibility and safety of early discharge after transfemoral transcatheter aortic valve implantation with the Edwards SAPIENXT prosthesis. Am J Cardiol. 2015 Apr 15;115(8):1116-22.

[8] Wood DA, Lauck SB, Cairns JA, Humphries KH, Cook R, Welsh R, et al. The Vancouver 3M (Multidisciplinary, Multimodality, But Minimalist) Clinical Pathway Facilitates Safe Next-Day Discharge Home at Low-, Medium-, and High-Volume Transfemoral Transcatheter Aortic Valve Replacement Centers: The 3M TAVR Study. JACC Cardiovasc Interv. 2019 Mar 11;12(5):459-69.

[9] Tchetche D, de Biase C, Brochado B, Mastrokostopoulos A. How to Make the TAVI Pathway More Efficient. Interv Cardiol. 2019 Feb;14(1):31-3.

[10] Rodés-Cabau J, Webb JG, Cheung A, Ye J, Dumont E, Feindel CM, et al. Transcatheter aortic valve implantation for the treatment of severe symptomatic aortic stenosis in patients at very high or prohibitive surgical risk: acute and late outcomes of the multicenter Canadian experience. J Am Coll Cardiol. 2010 Mar 16;55(11):1080-90.

[11] Auffret V, Lefevre T, Van Belle E, Eltchaninoff $H$, lung B, Koning R, et al. Temporal Trends in Transcatheter Aortic Valve Replacement in France: FRANCE 2 to FRANCE TAVI. J Am Coll Cardiol. 2017 Jul 4;70(1):42-55.

[12] Barbash IM, Barbanti M, Webb J, Molina-Martin De Nicolas J, Abramowitz Y, Latib A, et al. Comparison of vascular closure devices for access site closure after transfemoral aortic valve implantation. Eur Heart J. 2015 Dec 14;36(47):3370-9.

[13] De Palma R, Settergren M, Rück A, Linder R, Saleh N. Impact of percutaneous femoral arteriotomy closure using the MANTATM device on vascular and bleeding complications after transcatheter aortic valve replacement. Catheter Cardiovasc Interv. 2018 Mar 25

[14] Allende R, Urena M, Cordoba JG, Ribeiro HB, Amat-Santos I, DeLarochellière R, et al. Impact of the use of transradial versus transfemoral approach as secondary access in transcatheter aortic valve implantation procedures. Am J Cardiol. 2014 Dec 1;114(11):1729-34.

[15] Wynne DG, Rampat R, Trivedi U, de Belder A, Hill A, Hutchinson N, et al. Transradial Secondary Arterial Access for Transcatheter Aortic Valve Implantation: Experience and Limitations. Heart Lung Circ. 2015 Jul;24(7):682-5.

[16] Jolly SS, Amlani S, Hamon M, Yusuf S, Mehta SR. Radial versus femoral access for coronary angiography or intervention and the impact on major bleeding and ischemic events: a systematic review and meta-analysis of randomized trials. Am Heart J. 2009 Jan;157(1):132-40. 
[17] Grube E, Naber C, Abizaid A, Sousa E, Mendiz O, Lemos P, et al. Feasibility of transcatheter aortic valve implantation without balloon pre-dilation: a pilot study. JACC Cardiovasc Interv. 2011 Jul;4(7):751-7.

[18] Fiorina C, Maffeo D, Curello S, Lipartiti F, Chizzola G, D'Aloia A, et al. Direct transcatheter aortic valve implantation with self-expandable bioprosthesis: feasibility and safety. Cardiovasc Revasc Med. 2014 Jun;15(4):200-3.

[19] Spaziano M, Sawaya F, Chevalier B, Roy A, Neylon A, Garot P, et al. Comparison of Systematic Predilation, Selective Predilation, and Direct Transcatheter Aortic Valve Implantation With the SAPIEN S3 Valve. Can J Cardiol. 2017 Feb;33(2):260-8.

[20] Deharo P, Jaussaud N, Grisoli D, Camus O, Resseguier N, Le Breton H, et al. Impact of Direct Transcatheter Aortic Valve Replacement Without Balloon Aortic Valvuloplasty on Procedural and Clinical Outcomes: Insights From the FRANCE TAVI Registry. JACC Cardiovasc Interv. 2018 Oct 8;11(19):1956-65.

[21] Faurie B, Abdellaoui M, Wautot F, Staat $P$, Champagnac D, Wintzer-Wehekind J, et al. Rapid pacing using the left ventricular guidewire: Reviving an old technique to simplify BAV and TAVI procedures. Cathet Cardiovasc Intervent. 2016 Nov 15;88(6):988-93.

[22] Leclercq F, lemmi A, Lattuca B, Macia J-C, Gervasoni R, Roubille F, et al. Feasibility and Safety of Transcatheter Aortic Valve Implantation Performed Without Intensive Care Unit Admission. Am J Cardiol. 2016 Jul 1;118(1):99-106.

[23] Durand E, Borz B, Godin M, Tron C, Litzler P-Y, Bessou J-P, et al. Transfemoral Aortic Valve Replacement With the Edwards SAPIEN and Edwards SAPIEN XT Prosthesis Using Exclusively Local Anesthesia and Fluoroscopic Guidance: Feasibility and 30-Day Outcomes. JACC: Cardiovascular Interventions. 2012 May 1;5(5):461-7.

[24] Toggweiler S, Stortecky S, Holy E, Zuk K, Cuculi F, Nietlispach F, et al. The Electrocardiogram After Transcatheter Aortic Valve Replacement Determines the Risk for Post-Procedural High-Degree AV Block and the Need for Telemetry Monitoring. JACC Cardiovasc Interv. 2016 27;9(12):1269-76.

[25] Barbanti M, van Mourik MS, Spence MS, Icovelli F, Martinelli GL, Muir DF, et al. Optimising patient discharge management after transfemoral transcatheter aortic valve implantation: the multicentre European FAST-TAVI trial. Eurolntervention. 2019 Jun 20;15(2):147-54.

[26] Oguri A, Yamamoto M, Mouillet G, Gilard M, Laskar M, Eltchaninoff H, et al. Clinical outcomes and safety of transfemoral aortic valve implantation under general versus local anesthesia: subanalysis of the French Aortic National CoreValve and Edwards 2 registry. Circ Cardiovasc Interv. 2014 Aug;7(4):602-10.

[27] Hosoba S, Yamamoto M, Shioda K, Sago M, Koyama Y, Shimura T, et al. Safety and efficacy of minimalist approach in transfemoral transcatheter aortic valve replacement: insights from the Optimized transCathEter vAlvular interventioN-Transcatheter Aortic Valve Implantation (OCEAN-TAVI) registry. Interact Cardiovasc Thorac Surg. 2017 Oct 31;

[28] Villablanca PA, Mohananey D, Nikolic K, Bangalore S, Slovut DP, Mathew V, et al. Comparison of local versus general anesthesia in patients undergoing transcatheter aortic valve replacement: A metaanalysis. Catheter Cardiovasc Interv. 2017 Jul 24

[29] Ehret C, Rossaint R, Foldenauer AC, Stoppe C, Stevanovic A, Dohms K, et al. Is local anaesthesia a favourable approach for transcatheter aortic valve implantation? A systematic review and metaanalysis comparing local and general anaesthesia. BMJ Open. 2017 Sep 1;7(9):e016321.

[30] Leclercq F, Akodad M, Macia J-C, Gandet T, Lattuca B, Schmutz L, et al. Vascular Complications and Bleeding After Transfemoral Transcatheter Aortic Valve Implantation Performed Through Open Surgical Access. Am J Cardiol. 2015 Nov 1;116(9):1399-404.

[31] Kadakia MB, Herrmann HC, Desai ND, Fox Z, Ogbara J, Anwaruddin S, et al. Factors associated with vascular complications in patients undergoing balloon-expandable transfemoral transcatheter aortic valve replacement via open versus percutaneous approaches. Circ Cardiovasc Interv. 2014 Aug;7(4):570-6. 
[32]Toutouzas K, Benetos G, Voudris V, Drakopoulou M, Stathogiannis K, Latsios G, et al. Pre-Dilatation Versus No Pre-Dilatation for Implantation of a Self-Expanding Valve in All Comers Undergoing TAVR: The DIRECT Trial. JACC Cardiovasc Interv. 2019 Apr 22;12(8):767-77

[33] Leclercq F, Robert P, Labour J, Lattuca B, Akodad M, Macia J-C, et al. Prior balloon valvuloplasty versus DIRECT transcatheter Aortic Valve Implantation (DIRECTAVI): study protocol for a randomized controlled trial. Trials. $2017 \mathrm{Jul}$ 4;18(1):303.

[34] Mallikethi-Reddy S, Akintoye E, Telila T, Sudhakar R, Jagadeesh K, Briasoulis A, et al. Transcatheter aortic valve implantation in the United States: Predictors of early hospital discharge. J Interv Cardiol. 2017 Apr;30(2):149-55. 
Légende des figures

Figure 1 : Profil type du patient pouvant bénéficier d'une approche simplifiée

Figure 2: Composition de la "Heart Team » avec décision à l'issue de staff pluridisciplinaire de la prise en charge du rétrécissement aortique

Figure 3: Eléments de simplification de la procédure TAVI en per et post-procédure 


\section{Pré-procédure}

\section{Per et post-procédure}

Autonomie et entourage présent

Abord transfémoral favorable

Absence de comorbidités majeures

Absence de bloc de branche droit
Absence de complication

Bon résultat de la procédure

ECG non modifié

Succès de lever précoce 
Cardiologue non invasif interventionnel

Coordinateur*

Anesthésiste

\section{Patient}

Chirurgien cardiaque
Cardiologue référent

\section{Gériatre}



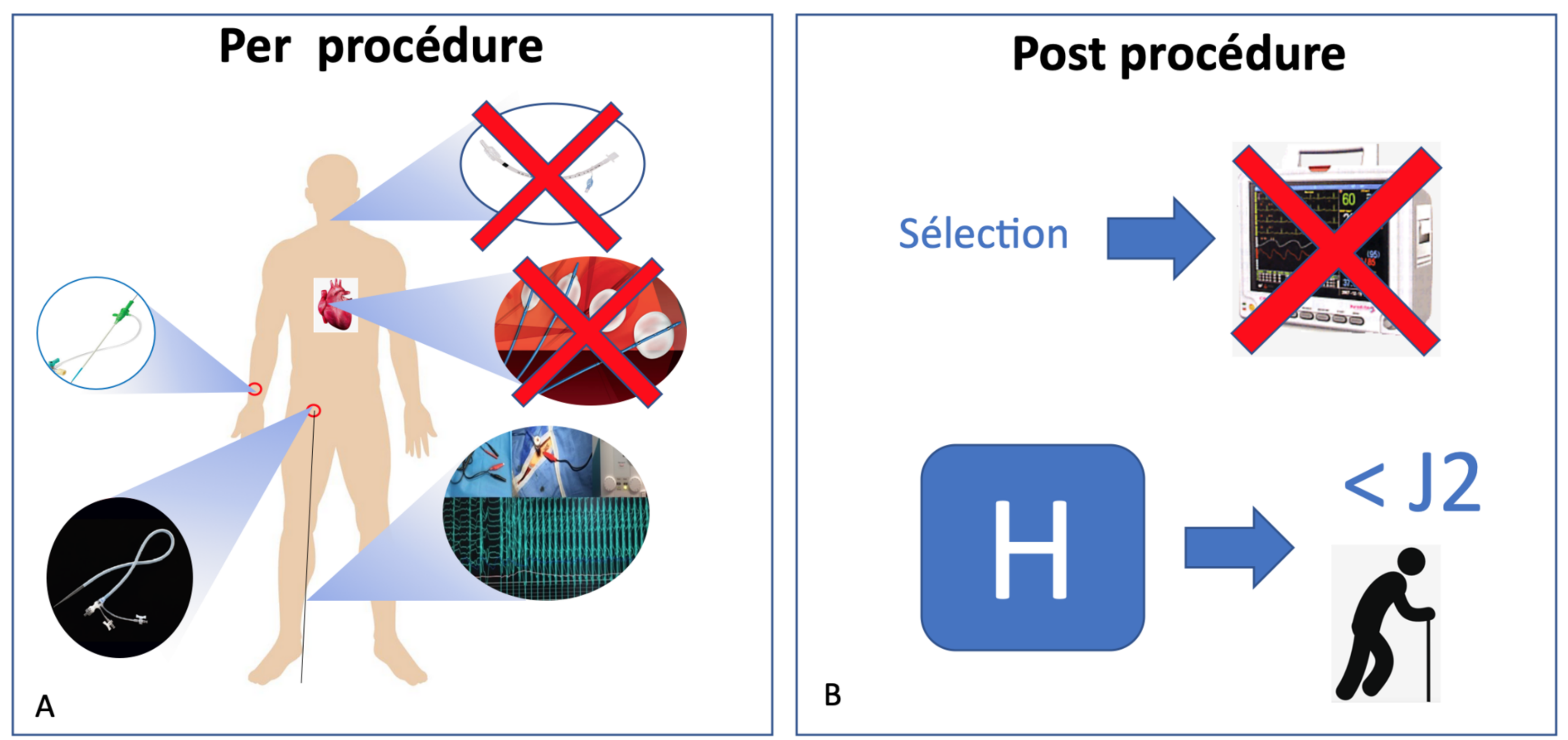